\title{
Senescence: Still an Unsolved Problem of Biology
}

\author{
Mark Roper ${ }^{1}$, Pol Capdevila ${ }^{1}$, and Roberto Salguero-Gomez ${ }^{2}$ \\ ${ }^{1}$ Oxford University \\ ${ }^{2}$ University of Oxford
}

May 5, 2020

\begin{abstract}
Despite ca. seven decades of theoretical elaboration since Peter Medawar's foundational 'An Unsolved Problem of Biology', we argue that the fundamental problem of the evolution of senescence, i.e. the increasing risk of mortality and decline in reproduction with age after maturity, remains unsolved. Theories of senescence predict the inescapability of senescence, or its universality at least among species with a clear germ-soma barrier. Here, using demographic information for 475 multicellular species, we exemplify the discrepancy between these theoretical predictions and currently available data. We derive age-based trajectories of mortality and reproduction whose form cannot be satisfactorily explained by the theories of senescence, and show that species' may often display senescence for one fitness component but not the other. We propose that theories of senescence must be extended beyond merely individual chronological age; size, the species' ecological context, and kin selection may all play hidden, yet integral roles in shaping patterns of senescence.
\end{abstract}

\section{Hosted file}

Still an Unsolved Problem of Biology_EL_F.pdf available at https://authorea.com/users/294340/ articles/433563-senescence-still-an-unsolved-problem-of-biology 